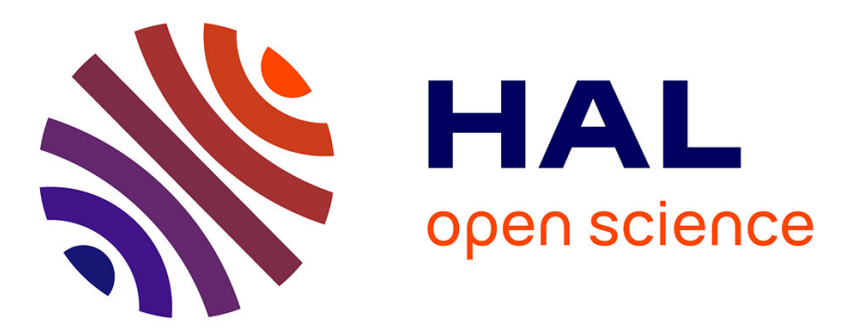

\title{
A new accurate numerical method of approximation of chaotic solutions of dynamical model equations with quadratic nonlinearities
}

\author{
René Lozi, Vasily A. Pogonin, Alexander N. Pchelintsev
}

\section{- To cite this version:}

René Lozi, Vasily A. Pogonin, Alexander N. Pchelintsev. A new accurate numerical method of approximation of chaotic solutions of dynamical model equations with quadratic nonlinearities. Chaos, Solitons \& Fractals, 2016, 91, pp.108-114. 10.1016/j.chaos.2016.05.010 . hal-01319597

\author{
HAL Id: hal-01319597 \\ https://hal.science/hal-01319597
}

Submitted on 21 May 2016

HAL is a multi-disciplinary open access archive for the deposit and dissemination of scientific research documents, whether they are published or not. The documents may come from teaching and research institutions in France or abroad, or from public or private research centers.
L'archive ouverte pluridisciplinaire HAL, est destinée au dépôt et à la diffusion de documents scientifiques de niveau recherche, publiés ou non, émanant des établissements d'enseignement et de recherche français ou étrangers, des laboratoires publics ou privés. 


\title{
A New Accurate Numerical Method of Approximation of Chaotic Solutions of Dynamical Model Equations with Quadratic Nonlinearities
}

\author{
René Lozi ${ }^{\mathrm{a}, *}$, Vasiliy A. Pogonin ${ }^{\mathrm{b}}$, Alexander N. Pchelintsev ${ }^{\mathrm{c}, *}$ \\ ${ }^{a}$ University of Nice-Sophia Antipolis, Nice, U.C.A., UMR CNRS 7351, France \\ ${ }^{b}$ Tambov State Technical University, ul. Sovetskaya 106, Tambov, 392000, Russia \\ ${ }^{c}$ Tambov State Technical University, ul. Sovetskaya 106, Tambov, 392000, Russia
}

\begin{abstract}
In this article the authors describe the method of construction of approximate chaotic solutions of dynamical model equations with quadratic nonlinearities in a general form using a new accurate numerical method. Numerous systems of chaotic dynamical systems of this type are studied in modern literature. The authors find the region of convergence of the method and offer an algorithm of construction and several criteria to check the accuracy of the approximate chaotic solutions.
\end{abstract}

Keywords: attractor, Lorenz system, Chen system, Nose-Hoover oscillator, power series, region of convergence, almost periodic function.

2000 MSC: 34D45, 37D45, 65G20, 65G50, 65L05, 65L07, 65L20, 65P20,

$65 \mathrm{~L} 70$.

\section{Introduction}

Chaotic dynamical systems are difficult to analyse. A close formula giving the solution has not been found yet. Therefore numerical approximations are mandatory in order to follow the motion of a particle driven by a system of a nonlinear differential equation.

\footnotetext{
*Corresponding authors

Email addresses: rlozi@unice.fr (René Lozi), pogvas@inbox.ru (Vasiliy A. Pogonin), pchelintsev.an@yandex.ru (Alexander N. Pchelintsev)
} 
Let us consider the system of a differential equations with quadratic nonlinearity

$$
\dot{x}=B_{0}+B_{1} x+\varphi(x),
$$

where $x(t)=\left[x_{(1)}(t) \ldots x_{(m)}(t)\right]^{\mathrm{T}}$ is a $m$-dimensional real vector function, $B_{0}$ is a given $m$-dimensional real column vector,

$$
\varphi(x)=\left[\varphi_{(1)}(x) \ldots \varphi_{(m)}(x)\right]^{\mathrm{T}},
$$

$\varphi_{(p)}(x)=\left\langle Q_{p} x, x\right\rangle, B_{1}$ and $Q_{p}(p=\overline{1, m})$ are given real $(m \times m)$ matrices.

Suppose that the system (1) has a bounded solution for $t \geq 0$. Thus, the corresponding trajectory is attracted to the limit trajectory, see [1, pp. 338340]. Hence, this trajectory determines the behavior of the bounded solutions of the system (1) when time goes to infinity. The limiting trajectory can be a point of equilibrium, a cycle, or can be described by an almost periodic function or can have a more complicated structure such as a strange attractor.

For some dynamical systems (1) the solutions are unstable on their attractors. It causes difficulties applying numerical methods for solving corresponding systems of ordinary differential equations (ODE). The problem is not limited to ODE with entire derivatives, but also for dynamical systems governed by fractional derivatives [2, 3, 4]. Many researchers use different numerical schemes based on classical methods, e.g. the explicit Euler scheme with the central-difference scheme [5], the Adams scheme [6], the higher derivatives scheme [7], the 4th order Runge-Kutta method [8] and the second-order accurate Adams-Bashforth method [9] for the Lorenz system. However, the above methods cannot be used for (1) due to the instability of chaotic solutions, since the global error increases with the size of the integration interval [10, 11]. Strogatz [12, pp. 320-323] computes the estimate of the time $T_{c}$ when the trajectories of the system (1) decouple critically for the Lorenz system. In [11] the authors present the regression dependence to estimate $T_{c}$ for the integration step $\Delta t$ and the order $N_{o}$ of the numerical scheme

$$
T_{c}\left(N_{o}, \Delta t\right) \approx-2.6 N_{o} \lg \Delta t
$$

for the classical values of parameters of the Lorenz system. They also highlights that the numerical solution converges to different positions of equilibria for various values $\Delta t$ for the transient chaotic behavior.

Most importantly, the result cannot be improved by decreasing the integration step $\Delta t$, since the integration error has an extremum as a function 
of $\Delta t$. This problem can be solved by using high-accuracy calculations [13]. However, this approach restricts the study: on the one hand, the way to decrease the error is narrow (to change $\Delta t$ and the accuracy of real number representation in order to control the calculation process); on the other hand, the number of operations needed for very small $\Delta t$ is large. The Runge-Kutta methods can be applied to obtain solutions with a higher accuracy, but the corresponding formulas for $N_{o}>6$ are extremely cumbersome [14, 15].

In [16] the authors present the multistage spectral relaxation method (MSRM) which differs from the previous direct methods. They use the Chebyshev spectral method to solve the system (1) in the Gauss-Siedel form by an iteration scheme at each subinterval of integration. The advantage is that the accumulation of errors is not as great as it was in the direct methods. Motsa et al. compare the numerical results of MSRM with the piecewise successive linearization method [17]. However, the authors do not study the error of the method as an independent unit and increase the risk of rounding errors.

To find approximate solutions of systems of differential equations, the method of power series (or the method of Taylor series) is sometimes used. In [18, 19, 20, this method is used as the Adomian decomposition method $(\mathrm{ADM})$. In those studies, the authors obtain the coefficients of expansion of the solution in a power series for different systems of the form (11) without finding the radius of convergence. The error of the approximate chaotic solution is only compared with the numerical results using the Runge-Kutta methods. Vadasz and Olek [21] also study the dependence of ratio of coefficients of power series with respect to the number of terms in the series.

In this article we consider a modification of the power series method (similar to ADM) for the system (11). An advantage over the general scheme of the Taylor series method is that the expansion coefficients can be rapidly calculated by formulas in comparison to the procedure of symbolic differentiation of the right-hand sides of the system equations (in the nonlinear case huge memory is needed to store the symbol expressions in the calculation of the higher-order derivatives). Also, an estimate of the region of convergence of the power series is obtained, and some criteria for checking the accuracy of the approximate chaotic solutions is given in this article. Recently such an approach has been applied to the Lorenz and Chen systems [22, 23]. Here, we generalize our results for the systems in the form (1). 


\section{Some Examples of Chaotic Systems with Quadratic Nonlinear- ities}

In this section, we give several examples found in the literature on chaotic systems of the form (1), for which our method can be applied.

\subsection{The well known Lorenz system}

$$
\left\{\begin{array}{l}
\dot{x}_{(1)}=\sigma\left(x_{(2)}-x_{(1)}\right) \\
\dot{x}_{(2)}=r x_{(1)}-x_{(2)}-x_{(1)} x_{(3)}, \\
\dot{x}_{(3)}=x_{(1)} x_{(2)}-b x_{(3)} .
\end{array}\right.
$$

For this system, the matrices are

$$
\begin{gathered}
B_{0}=\mathbf{0}, \quad B_{1}=\left[\begin{array}{ccc}
-\sigma & \sigma & 0 \\
r & -1 & 0 \\
0 & 0 & -b
\end{array}\right], Q_{1}=\mathbf{0}, \quad Q_{2}=\left[\begin{array}{ccc}
0 & 0 & -1 \\
0 & 0 & 0 \\
0 & 0 & 0
\end{array}\right] \\
Q_{3}=\left[\begin{array}{lll}
0 & 1 & 0 \\
0 & 0 & 0 \\
0 & 0 & 0
\end{array}\right]
\end{gathered}
$$

2.2. The Chen system [24, 25]

$$
\left\{\begin{array}{l}
\dot{x}_{(1)}=a\left(x_{(2)}-x_{(1)}\right) \\
\dot{x}_{(2)}=(c-a) x_{(1)}-x_{(1)} x_{(3)}+c x_{(2)}, \\
\dot{x}_{(3)}=x_{(1)} x_{(2)}-b x_{(3)}
\end{array}\right.
$$

for which the matrices are

$$
\begin{gathered}
B_{0}=\mathbf{0}, \quad B_{1}=\left[\begin{array}{ccc}
-a & a & 0 \\
c-a & c & 0 \\
0 & 0 & -b
\end{array}\right], \quad Q_{1}=\mathbf{0}, \quad Q_{2}=\left[\begin{array}{ccc}
0 & 0 & -1 \\
0 & 0 & 0 \\
0 & 0 & 0
\end{array}\right] \\
Q_{3}=\left[\begin{array}{lll}
0 & 1 & 0 \\
0 & 0 & 0 \\
0 & 0 & 0
\end{array}\right]
\end{gathered}
$$


2.3. The Nose-Hoover oscillator [26]

$$
\left\{\begin{array}{l}
\dot{x}_{(1)}=x_{(2)} \\
\dot{x}_{(2)}=-x_{(1)}-x_{(2)} x_{(3)} \\
\dot{x}_{(3)}=\left(x_{(2)}^{2}-1\right) / q .
\end{array}\right.
$$

In this case, the matrices are

$$
\begin{gathered}
B_{0}=\left[\begin{array}{c}
0 \\
0 \\
-1 / q
\end{array}\right], B_{1}=\left[\begin{array}{rrr}
0 & 1 & 0 \\
-1 & 0 & 0 \\
0 & 0 & 0
\end{array}\right], Q_{1}=\mathbf{0}, Q_{2}=\left[\begin{array}{rrr}
0 & 0 & 0 \\
0 & 0 & -1 \\
0 & 0 & 0
\end{array}\right] \\
Q_{3}=\left[\begin{array}{rrr}
0 & 0 & 0 \\
0 & 1 / q & 0 \\
0 & 0 & 0
\end{array}\right]
\end{gathered}
$$

2.4. The Sprott-Jafari system 27]

(we study this example in Sec. 7 to show the efficiency of our method)

$$
\left\{\begin{array}{l}
\dot{x}_{(1)}=x_{(2)} \\
\dot{x}_{(2)}=-x_{(1)}+x_{(2)} x_{(3)} \\
\dot{x}_{(3)}=x_{(3)}+a x_{(1)}^{2}-x_{(2)}^{2}-b
\end{array}\right.
$$

with corresponding matrices

$$
\begin{gathered}
B_{0}=\left[\begin{array}{c}
0 \\
0 \\
-b
\end{array}\right], B_{1}=\left[\begin{array}{rrr}
0 & 1 & 0 \\
-1 & 0 & 0 \\
0 & 0 & 1
\end{array}\right], Q_{1}=\mathbf{0}, Q_{2}=\left[\begin{array}{lll}
0 & 0 & 0 \\
0 & 0 & 1 \\
0 & 0 & 0
\end{array}\right], \\
Q_{3}=\left[\begin{array}{rrr}
a & 0 & 0 \\
0 & -1 & 0 \\
0 & 0 & 0
\end{array}\right] .
\end{gathered}
$$

In 28] Sprott lists the nineteen three-dimensional ODEs of the form (1). 
2.5. Increasing the size of the system, one finds the 4D Rössler model [29]

$$
\left\{\begin{array}{l}
\dot{x}_{(1)}=-\left(x_{(2)}+x_{(3)}\right), \\
\dot{x}_{(2)}=x_{(1)}+a x_{(2)}+x_{(4)}, \\
\dot{x}_{(3)}=b+x_{(1)} x_{(3)}, \\
\dot{x}_{(4)}=-c x_{(3)}+d x_{(4)} .
\end{array}\right.
$$

In this case, the matrices are

$$
\begin{gathered}
B_{0}=\left[\begin{array}{l}
0 \\
0 \\
b \\
0
\end{array}\right], B_{1}=\left[\begin{array}{rrrr}
0 & -1 & -1 & 0 \\
1 & a & 0 & 1 \\
0 & 0 & 0 & 0 \\
0 & 0 & -c & d
\end{array}\right], Q_{1}=Q_{2}=Q_{4}=\mathbf{0}, \\
Q_{3}=\left[\begin{array}{llll}
0 & 0 & 1 & 0 \\
0 & 0 & 0 & 0 \\
0 & 0 & 0 & 0 \\
0 & 0 & 0 & 0
\end{array}\right] .
\end{gathered}
$$

2.6. Finally, our method is even valid for the nine-dimensional Lorenz system [30]

$$
\left\{\begin{array}{l}
\dot{x}_{(1)}=-\sigma b_{1} x_{(1)}-\sigma b_{2} x_{(7)}-x_{(2)} x_{(4)}+b_{4} x_{(4)}^{2}+b_{3} x_{(3)} x_{(5)}, \\
\dot{x}_{(2)}=-\sigma x_{(2)}-\frac{\sigma}{2} x_{(9)}+x_{(1)} x_{(4)}-x_{(2)} x_{(5)}+x_{(4)} x_{(5)}, \\
\dot{x}_{(3)}=-\sigma b_{1} x_{(3)}+\sigma b_{2} x_{(8)}+x_{(2)} x_{(4)}-b_{4} x_{(2)}^{2}-b_{3} x_{(1)} x_{(5)}, \\
\dot{x}_{(4)}=-\sigma x_{(4)}+\frac{\sigma}{2} x_{(9)}-x_{(2)} x_{(3)}-x_{(2)} x_{(5)}+x_{(4)} x_{(5)}, \\
\dot{x}_{(5)}=-\sigma b_{5} x_{(5)}+\frac{1}{2} x_{(2)}^{2}-\frac{1}{2} x_{(4)}^{2}, \\
\dot{x}_{(6)}=-b_{6} x_{(6)}+x_{(2)} x_{(9)}-x_{(4)} x_{(9)}, \\
\dot{x}_{(7)}=-r x_{(1)}-b_{1} x_{(7)}+2 x_{(5)} x_{(8)}-x_{(4)} x_{(9)}, \\
\dot{x}_{(8)}=r x_{(3)}-b_{1} x_{(8)}-2 x_{(5)} x_{(7)}+x_{(2)} x_{(9)}, \\
\dot{x}_{(9)}=-r x_{(2)}+r x_{(4)}-x_{(9)}-2 x_{(2)} x_{(6)}+2 x_{(4)} x_{(6)}+x_{(4)} x_{(7)}-x_{(2)} x_{(8)} .
\end{array}\right.
$$




\section{The Numeric-Analytical Solution of Differential Equations}

The system (1) has a polynomial right-hand side. This allows the use of an explicit formula to calculate the power series coefficients and estimate the region of convergence.

Let

$$
x(t)=\sum_{i=0}^{\infty} \Lambda_{i} t^{i},
$$

where $x(0)=\Lambda_{0}$ is an initial condition for the system (1), $\Lambda_{i} \in \mathbb{R}^{m}$. The multiplication of the power series in the Cauchy form in the vector notation is

$$
\varphi_{(p)}(x)=\sum_{i=0}^{\infty} \Phi_{i(p)} t^{i}, \quad \Phi_{i(p)}=\sum_{j=0}^{i}\left\langle Q_{p} \Lambda_{j}, \Lambda_{i-j}\right\rangle, \quad p=\overline{1, m} .
$$

Let

$$
\Phi_{i}=\left[\begin{array}{lll}
\Phi_{i(1)} & \ldots & \Phi_{i(m)}
\end{array}\right]^{\mathrm{T}} .
$$

Note that

$$
\Lambda_{1}=B_{0}+B_{1} \Lambda_{0}+\Phi_{0} .
$$

Equating the coefficients at the same powers, we obtain from the system (1) the recurrence relation to calculate the power series coefficients in (4) for $i \geq 2$

$$
\Lambda_{i}=\frac{B_{1} \Lambda_{i-1}+\Phi_{i-1}}{i} .
$$

This form of coefficients of the power series is simpler and faster to compute than in ADM (because it does not contain factorials).

Although a right-hand side of the system (11) is analytical everywhere, the radius of convergence of the series can be bounded and it depends on $\Lambda_{0}$. Therefore, only a part of the trajectory can be obtained by the above method. The procedure of constructing the trajectory arc of any time length is given by joining several parts of the trajectory computed by our method. These parts of trajectory describe the desired solution, when the series (4) converges. The integration error is accumulated when passing from one trajectory part to another part (due to the error of finding the current approximate solution), however it can be controlled by varying the accuracy of power series expansion. Here, highly accurate calculations may help in continuing the solution for very large time intervals because the accuracy $\varepsilon_{p}$ of power series expansion cannot be less than the machine epsilon $\varepsilon_{m}$. 


\section{Algorithm for Constructing the Trajectory Arc}

Let us consider the algorithm of constructing the trajectory arc for the time interval $[0 ; T]$, where the value $T$ is given:

\section{begin}

1. Set the values of $\varepsilon_{m}$ and way;

2. Input the values of $T, \varepsilon_{p}$ and $\Lambda_{0}$;

3. $t:=0$

4. Calculate the value of $\Delta t$ from a function of $\Lambda_{0}$;

5. $t:=t+\Delta t$

6. If $t>T$ then flag $:=1 ; \Delta t:=\Delta t-(t-T)$

Else If $t<T$ then flag $:=0$

Else flag $:=1$;

7. $p:=1 ; i:=0$;

8. $x:=\Lambda_{0}$;

9. $i:=i+1 ; p:=p \cdot$ way $\cdot \Delta t$;

10. Calculate $\Lambda_{i}$ by the formulas (5) and (6);

11. $x:=x+\Lambda_{i} \cdot p$;

12. $L:=\left\|\Lambda_{i}\right\| \cdot|p|$;

13. If $L>\varepsilon_{p}$ then Goto Step 9;

14. $\Lambda_{0}:=x$

15. If $f l a g=0$ then Goto Step 4;

16. Print $\Lambda_{0}$

end.

The variable way provides an integration with reverse time when its value is -1 (for going forward way $=1$ ). For the sake of simplicity, the algorithm uses the positive values of time in both directions of motion along a trajectory. Thus, the algorithm allows the construction of an approximate solution in forward and backward time. We use both time directions to check the precision of the approximated solution and evenly increase such a precision.

Let us consider in detail the procedure of constructing the trajectory arc.

Let $t_{l} \in \Omega, l=\overline{1, N}$ be the index of the time intervals $\left[t_{l-1} ; t_{l}\right]$, where the series (4) converges, $N$ is the number of such intervals, $t_{0}=0, t_{N}=T$,

$$
\Omega=\left[t_{0} ; t_{1}\right] \cup\left[t_{1} ; t_{2}\right] \cup \ldots \cup\left[t_{N-1} ; t_{N}\right] .
$$


We define the vector $\Lambda_{0}$ of initial conditions for time $t_{0}$. Then the vectors $\Lambda_{i}$ $(i=1,2, \ldots)$ are calculated using the formulas (5) and (6) until the following estimate is not valid

$$
\left\|\Lambda_{i}\right\|\left|\Delta t_{l}\right|^{i}<\varepsilon_{p}
$$

where $\Delta t_{l}=t_{l}-t_{l-1}$. The module in (7) is used in case of negative values of the step $\Delta t_{l}$. Let $x_{1}(t)$ be the $m$-dimensional $n_{1}$-power polynomial, which is obtained from the estimation (7) in the first stage $(l=1)$ of calculation. In the second stage $(l=2)$ we set

$$
\Lambda_{0}:=x_{l-1}\left(\Delta t_{l-1}\right)
$$

and set the initial time $t_{1}$ to zero in order to simplify the calculations (since the system (1) is dynamic).

If $\tau_{l}$ is the region of convergence of the power series (4), then the value $\Delta t_{l}$ corresponds to

$$
0<\Delta t_{l}<\tau_{l}
$$

or

$$
-\tau_{l}<\Delta t_{l}<0
$$

\section{Estimating the Region of Convergence of the Power Series}

Estimating the region of convergence of the series (4) is important when approximate solutions of the system (1) are computed by the method described above. For this, we introduce the following notations:

$$
\begin{aligned}
& h_{1}\left(\Lambda_{0}\right)=\left\|\Lambda_{0}\right\|, \mu=m \max _{p=1, m}\left\|Q_{p}\right\|, \\
& h_{2}\left(\Lambda_{0}\right)=\left\{\begin{array}{l}
\left\|B_{0}\right\|+\left(\left\|B_{1}\right\|+2 \mu\right) h_{1}+\mu h_{1}^{2}, \text { if } h_{1}>1, \\
\left\|B_{0}\right\|+\left\|B_{1}\right\|+\mu \text { otherwise. }
\end{array}\right.
\end{aligned}
$$

Let us prove that the series (4) converges for $t \in\left(-\tau_{l} ; \tau_{l}\right)$, where $\tau_{l}=1 / h_{2}$. For this, the number $h_{2}$ (with $h_{2}|t|<1$ ) should be chosen such that

$$
\left\|\Lambda_{i} t^{i}\right\| \leq\left(h_{2}|t|\right)^{i}
$$

Then the series (4) converges absolutely by the direct comparison test. 
Theorem 1. The following inequalities hold

$$
\left\|\Lambda_{i}\right\| \leq h_{2}^{i}
$$

for any natural number $i$.

Proof. We use mathematical induction. Let us consider a case where $h_{1}>1$.

Let us show that (8) is valid for $i=1$. From the formula (5) and the Cauchy-Schwarz inequality, we have

$$
\begin{aligned}
& \left\|\Lambda_{1}\right\| \leq\left\|B_{0}\right\|+\left\|B_{1}\right\|\left\|\Lambda_{0}\right\|+m \max _{p=\overline{1, m}}\left|\left\langle Q_{p} \Lambda_{0}, \Lambda_{0}\right\rangle\right| \leq \\
& \leq\left\|B_{0}\right\|+\left\|B_{1}\right\| h_{1}+\mu h_{1}^{2} \leq h_{2}^{1},
\end{aligned}
$$

which proves that (8) is valid for $i=1$.

Assume that (8) is valid for $i=k$. Then it is also valid for any $j=\overline{1, k}$, that is,

$$
\left\|\Lambda_{j}\right\| \leq h_{2}^{j} .
$$

Let us prove that (8) is valid for $i=k+1$. Let us estimate

$$
\begin{aligned}
& \left\|\sum_{j=0}^{k}\left\langle Q_{p} \Lambda_{j}, \Lambda_{k-j}\right\rangle\right\| \leq \mu\left\|\Lambda_{0}\right\|\left\|\Lambda_{k}\right\|+\mu\left\|\Lambda_{k}\right\|\left\|\Lambda_{0}\right\|+ \\
& +\mu \sum_{j=1}^{k-1}\left\|\Lambda_{j}\right\|\left\|\Lambda_{k-j}\right\| \leq 2 \mu h_{1} h_{2}^{k}+ \\
& +\mu \sum_{j=1}^{k-1} h_{2}^{j} h_{2}^{k-j}=2 \mu h_{1} h_{2}^{k}+(k-1) \mu h_{2}^{k} .
\end{aligned}
$$

From the formula (6) and the inequalities (9) we obtain the estimate (considering that $k \geq 1$ and $h_{1}>1$ )

$$
\begin{aligned}
& \left\|\Lambda_{k+1}\right\| \leq \frac{\left\|B_{1}\right\|\left\|\Lambda_{k}\right\|+2 \mu h_{1} h_{2}^{k}}{k+1}+\frac{k-1}{k+1} \mu h_{2}^{k} \leq\left\|B_{1}\right\| h_{2}^{k}+ \\
& +2 \mu h_{1} h_{2}^{k}+\mu h_{2}^{k} \leq\left(\left\|B_{0}\right\|+\left\|B_{1}\right\|+2 \mu h_{1}+\mu\right) h_{2}^{k} \leq \\
& \leq h_{2} h_{2}^{k}=h_{2}^{k+1},
\end{aligned}
$$

which proves that (8) is valid for any natural number $i$. 
Now, let us consider the other case $h_{2} \leq 1$. Let us prove by induction that (8) is valid in this case. For $i=1$ we have

$$
\left\|\Lambda_{1}\right\| \leq\left\|B_{0}\right\|+\left\|B_{1}\right\|+\mu=h_{2}^{1} .
$$

Hence, when $i=1$, the statement (8) is valid.

Assume that (8) is valid for $i=k$. Let us estimate

$$
\left\|\sum_{j=0}^{k}\left\langle Q_{p} \Lambda_{j}, \Lambda_{k-j}\right\rangle\right\| \leq 2 \mu h_{2}^{k}+(k-1) \mu h_{2}^{k}=(k+1) \mu h_{2}^{k} .
$$

Let us prove that (8) is valid for $i=k+1$. It follows from the formula (6) and the above assumption that

$$
\begin{aligned}
& \left\|\Lambda_{k+1}\right\| \leq \frac{\left\|B_{1}\right\|\left\|\Lambda_{k}\right\|}{k+1}+\mu h_{2}^{k} \leq\left\|B_{1}\right\| h_{2}^{k}+\mu h_{2}^{k}= \\
& =\left(\left\|B_{1}\right\|+\mu\right) h_{2}^{k} \leq h_{2} h_{2}^{k}=h_{2}^{k+1},
\end{aligned}
$$

which proves that (8) is valid for any natural $i$ when $h_{2} \leq 1$.

\section{The Accuracy of the Approximate Chaotic Solution}

We assume

$$
\begin{gathered}
n_{\max }^{\{\text {way }\}}=\max _{l} n_{l}^{\{w a y\}}, l_{\max }^{\{\text {way }\}}=\underset{l}{\operatorname{indmax}} n_{l}^{\{\text {way }\}}, \\
\Delta t_{\max }^{\{\text {way }\}}=\text { way } \cdot \max _{l}\left|\Delta t_{l}^{\{w a y\}}\right|, d_{\max }^{\{\text {way }\}}=\operatorname{indmax}_{l}\left|\Delta t_{l}^{\{\text {way }\}}\right|,
\end{gathered}
$$

where the variable $\{$ way $\}$ shows the direction of the time.

In this section, we give some criteria to check the accuracy of the approximate chaotic solutions:

1. The accuracy $\varepsilon_{a}$ of approximation for way $=1$. When the inequality (7) holds, we increase the powers $n_{l}^{\{1\}}$ of all polynomials $x_{l}^{\{1\}}(t)$ (getting other approximate solution) and check the distance $\delta_{a}$ between the approximate solutions in the time interval $[0 ; T]$. If $\delta_{a}>\varepsilon_{a}$ then we increase $n_{l}$. 
2. In the special case of the Lorenz system, we check (2) as

$$
T \ll T_{c}\left(\overline{n^{\{1\}}}, \overline{\Delta t^{\{1\}}}\right)
$$

where $\overline{n^{\{1\}}}$ is the mean power of polynomials $x_{l}^{\{1\}}(t)$ and $\overline{\Delta t^{\{1\}}}$ is the mean value of $\Delta t_{l}^{\{1\}}$.

3. Let $\varepsilon_{R}$ be the radius of the neighborhood of initial point, in which the approximate solution returns at backward by time. We choose the precision $\varepsilon_{p}$ so that the following inequality holds:

$$
\left\|x_{\widehat{N}}^{\{-1\}}\left(\Delta t_{\widehat{N}}^{\{-1\}}\right)-x(0)\right\|<\varepsilon_{R},
$$

where $\widehat{N}=N^{\{-1\}}$. We do not know the exact solution of the system (1) in the general case, but we know exactly its initial point. Therefore, we can decide how many digits must coincide with the digits of the initial point. This accuracy control of approximate chaotic solutions is a great improvement when compared to the classical numerical methods. One issue is that a large amount of computing is needed (due to the repetition of forward and backward trajectories computation). The solutions of the system (1) are generally strongly unstable in reverse time: they immediately diverge from the attractor, since in our calculations we are close to it but not exactly on it. However, we cannot always go back to the acceptable neighborhood of the initial point. If a value of the accuracy $\varepsilon_{p}$ is too big large, then the point of the trajectory will go to infinity when we compute backward due to strong unstability. In [22] $\varepsilon_{p}=10^{-50}$ for the Lorenz system, in [23] $\varepsilon_{p}=10^{-80}$ and $10^{-53}$ for the Chen system.

4. A check of an approximate solution configuration (in addition to the previous criterion). We calculate the numbers (10) which describe the approximate solutions. Further, we verify that (enough of coincidence of a several approximate equalities, an example is given in Sec. 7)

$$
\begin{gathered}
N \approx \widehat{N}, n_{\max }^{\{1\}} \approx n_{\max }^{\{-1\}}, t_{l_{\max }^{\{1\}}}+\left|t_{l_{\max }^{\{-1\}}}\right| \approx T, \\
d_{\max }^{\{1\}}+d_{\max }^{\{-1\}} \approx N, t_{d_{\max }^{\{1\}}}+\left|t_{d_{\max }^{\{-1\}}}\right| \approx T .
\end{gathered}
$$

The advantage here is that we control the accuracy comparing the values of arguments of approximate solutions in forward and backward 
time. Thus, we conclude that it is sufficient to use the third and fourth criteria of accuracy.

The authors of the article 31] introduced a transformation of the Chen system to the Lorenz system as

$$
x_{L}\left(t_{L}\right)=-\frac{x_{C}\left(t_{C}\right)}{c}, t_{L}=-c \cdot t_{C}
$$

where $x_{L}$ and $x_{C}$ are vectors of phase coordinates for the Lorenz system and Chen system, respectively; $t_{L}$ and $t_{C}$ are times in these systems, $c$ is a parameter of the Chen system. Chen is right in the article [32] that using such a transformation, the time will be reversed in the Lorenz system for $c>0$.

Since usually

$$
c>1
$$

then the division of $x_{C}$ by $c$ decreases the computed error for the Lorenz system. The presented algorithm allows going backward. Thus the transformation (12) can give an approximate solution of the Lorenz system in enlarged scale if one knows an approximate solution of the Chen system, if the inequality 13 is valid.

\section{Calculating Experiment}

We present the results of computations based on the above scheme for the system (3). The parameter values are $a=8.888, b=4$. We have

$$
\begin{gathered}
\left\|Q_{1}\right\|=0,\left\|Q_{2}\right\|=1,\left\|Q_{3}\right\|=a, \mu=3 a,\left\|B_{0}\right\|=b,\left\|B_{1}\right\|=1, \\
h_{1}=\left|\Lambda_{0(1)}\right|+\left|\Lambda_{0(2)}\right|+\left|\Lambda_{0(3)}\right|,
\end{gathered}
$$

where $\Lambda_{0(i)}$ is the $i$-coordinate of the vector $\Lambda_{0}$. The initial condition is

$$
\Lambda_{0}=\left[\begin{array}{lll}
0 & 3.9 & 0.7
\end{array}\right]^{\mathrm{T}} .
$$

as in [27]. We consider the solution of system (3) for $T=T_{1}=34, \varepsilon_{p}=10^{-15}$ and $\varepsilon_{m}=1.0842 \cdot 10^{-19}$ (the number of bits for the mantissa of a real number is 64$)$. In this case, $\varepsilon_{R}=10^{-5}$. We use the Euclidean norm in the inequality (7) to reduce the number of terms in approximating polynomials. 


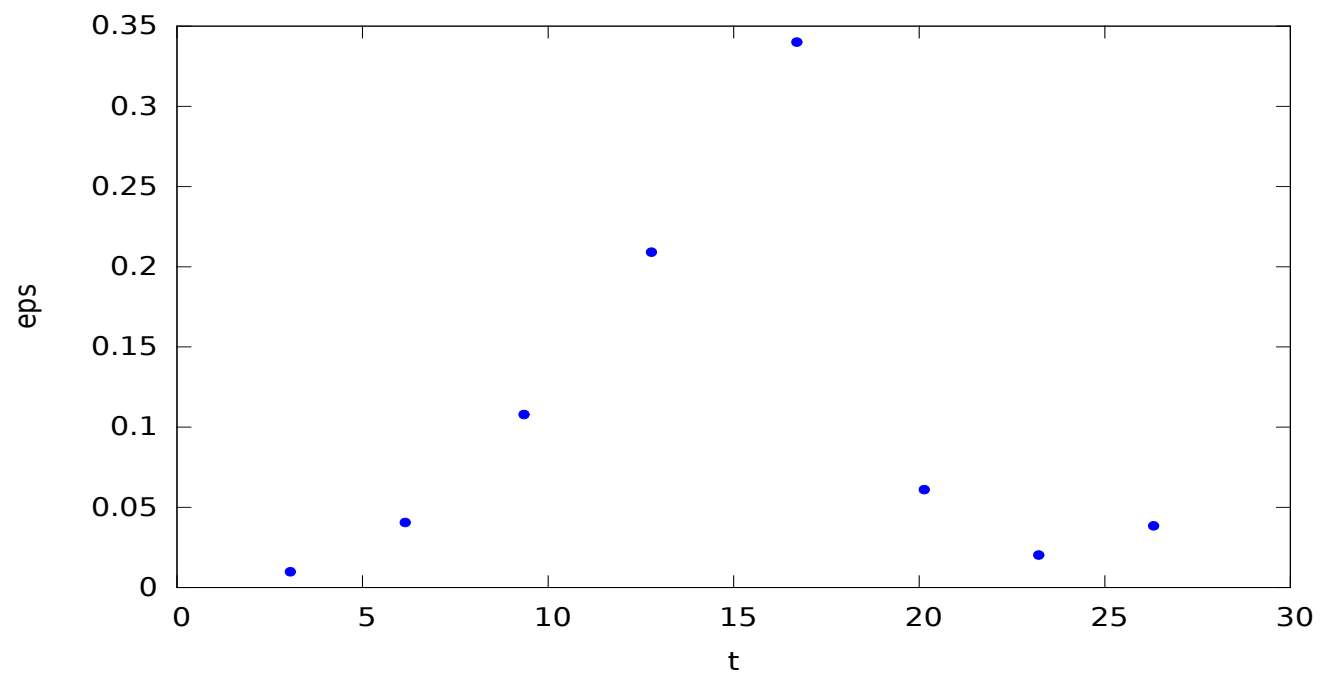

Figure 1: A graph of rapprochement of the trajectory with the initial point in the formula (14).

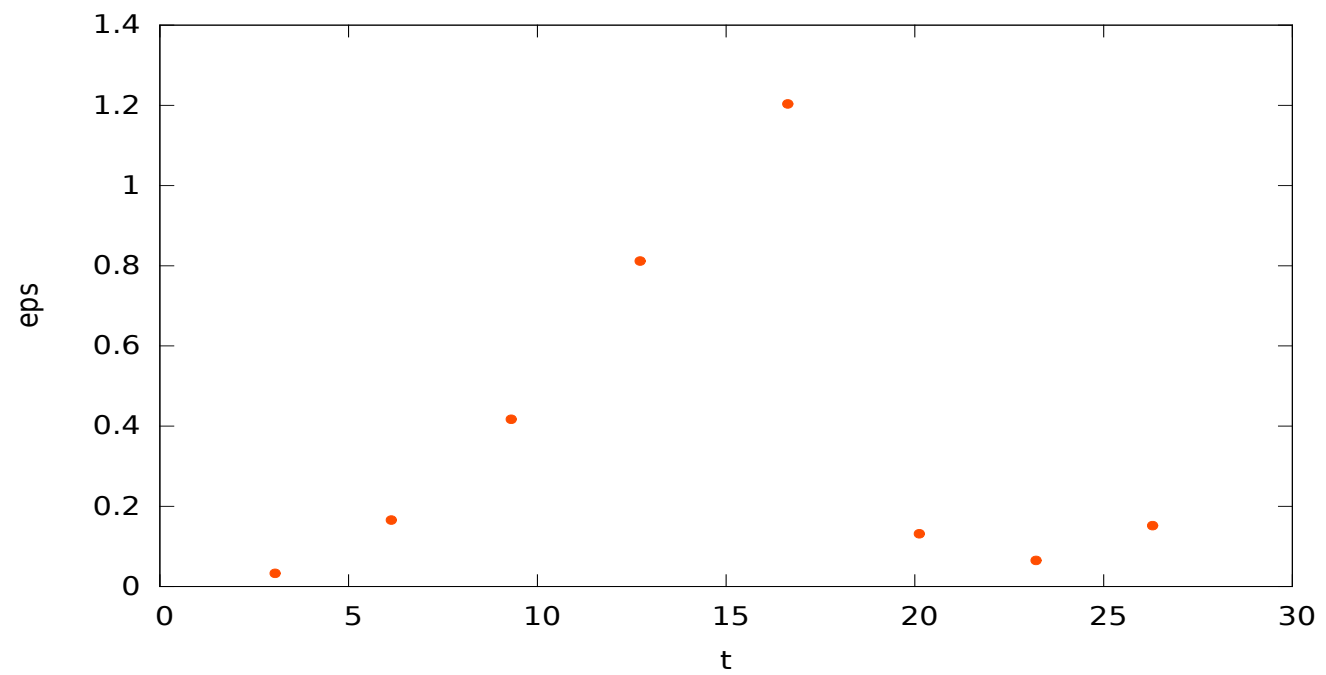

Figure 2: A graph of rapprochement of the trajectory with the initial point in the formula (15). 
Let us move the initial point by the corresponding trajectory at $T=T_{2}=$ 6 for a better approximation to the attractor. We get the point

$$
x_{N=15202}^{\{1\}}\left(T_{2}\right)=\left[\begin{array}{r}
-1.388360370340798916 \\
0.749102120590088463 \\
1.996651922943586555
\end{array}\right] .
$$

Then we set $\Lambda_{0}=x_{15202}^{\{1\}}\left(T_{2}\right)$.

Let us research the trajectory return in a neighborhood of the radius eps of the initial point because the limiting trajectory on the attractor has a property of recurrence. We construct a graph illustrating a rapprochement of the trajectory with the initial point (Fig. 1).

We move forward in time the initial point obtained from the above for $T=T_{3}=1$ by trajectory. We get the point

$$
x_{N=2210}^{\{1\}}\left(T_{3}\right)=\left[\begin{array}{r}
1.512058089397715359 \\
0.408816498647179974 \\
-4.158968175695369477
\end{array}\right] .
$$

Then we set $\Lambda_{0}=x_{2210}^{\{1\}}\left(T_{3}\right)$ again. The graph of rapprochement of the trajectory with the initial point is shown in Fig. 2.

The last point of the graph in Fig. 1 corresponds to the time

$$
t_{r_{1}}=26.316
$$

in Fig. 2

$$
t_{r_{2}}=26.297 \text {. }
$$

Note that

$$
\left\lceil T_{2}+\max \left\{t_{r_{1}}, T_{3}+t_{r_{2}}\right\}\right\rceil=T_{1} .
$$

We checked the fourth criteria in Sec. 6f for the initial point in (15) and $T=t_{r_{2}}$ (Tables 1 and 2). Note that the extrema can not be unique in the formulas (10). Therefore, not all of the approximate equalities (11) are valid.

The graphs in Figs. 1 and 2 show that the solution on an attractor of the system (3) is described by an almost periodic function because the moments of time of trajectory return to the neighborhood of the initial point are approximately at the same distance from each other. It shows the relative density of the set of such moments of time.

The trajectory arc constructed in the time interval $\left[0 ; t_{r_{1}}\right]$ is presented in Fig. 3. 


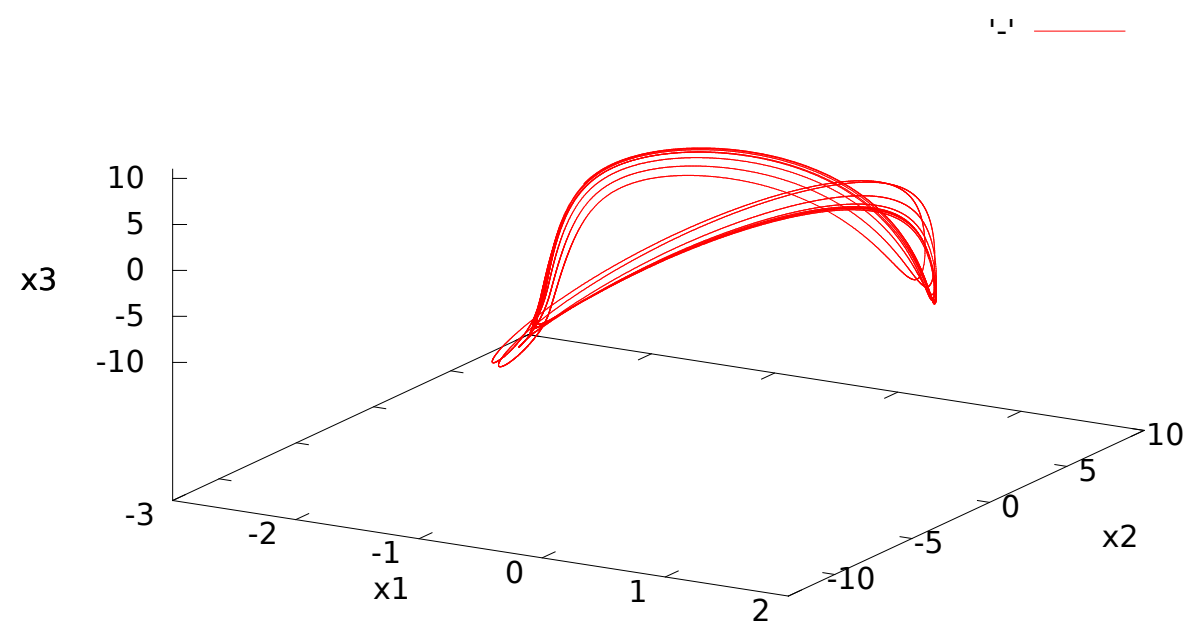

Figure 3: The trajectory arc constructed in the time interval $\left[0 ; t_{r_{1}}\right]$ for the initial point in (14).

Table 1: The results of calculating experiment for way $=1$.

\begin{tabular}{c|c}
\hline$N$ & 63244 \\
\hline$n_{\max }^{\{1\}}$ & 8 \\
\hline$l_{\max }^{\{1\}}$ & 61150 \\
\hline$t_{l_{\max }^{\{1\}}}$ & 25.1842 \\
\hline$d_{\max }^{\{1\}}$ & 13653 \\
\hline$t_{d_{\max }^{\{1\}}}$ & 6.40688 \\
\hline$\Delta t_{\max }^{\{1\}}$ & 0.00617846 \\
\hline
\end{tabular}


Table 2: The results of calculating experiment for way $=-1$.

\begin{tabular}{c|c}
\hline$\widehat{N}$ & 63244 \\
\hline$n_{\max }^{\{-1\}}$ & 8 \\
\hline$l_{\max }^{\{-1\}}$ & 2097 \\
\hline$t_{l_{\max }^{\{-1\}}}$ & -1.11196 \\
\hline$d_{\max }^{\{-1\}}$ & 13607 \\
\hline$t_{d_{\max }^{\{-1\}}}$ & -5.89213 \\
\hline$\Delta t_{\max }^{\{-1\}}$ & -0.0061386 \\
\hline
\end{tabular}

\section{Acknowledgements}

This article was partially supported by the Russian Science Foundation (Agreement No. 15-19-10028).

\section{References}

[1] V. V. Nemytskii, and V. V. Stepanov (1989), "Qualitative theory of differential equations", Dover Publications, New York, 523 pp.

[2] M.-S. Abdelouahab, R. Lozi, and L. Chua (2014), Memfractance: A Mathematical Paradigm for Circuit Elements with Memory // International Journal of Bifurcation and Chaos, Vol. 24, Iss. 9, 1430023, 29 pp.

doi:10.1142/S0218127414300237

[3] M.-S. Abdelouahab, and R. Lozi (2015), Hopf Bifurcation and Chaos in Simplest Fractional-Order Memristor-based Electrical Circuit // Indian Journal of Industrial and Applied Mathematics, Vol. 6, Iss. 2, pp. 105119.

doi:10.5958/1945-919X.2015.00009.2

[4] D. W. Brzezinski (2016), Accuracy Problems of Numerical Calculation of Fractional Order Derivatives and Integrals Applying the RiemannLiouville/Caputo Formulas // Applied Mathematics and Nonlinear Sciences, Vol. 1, Iss. 1, pp. 23-44. 
[5] E. N. Lorenz (1963), Deterministic Nonperiodic Flow // Journal of the Atmospheric Sciences, Vol. 20, Iss. 2, pp. 130-141. doi:10.1175/1520-0469(1963)020<0130:DNF>2.0.CO;2

[6] J. A. Yorke, and E. D. Yorke (1979), Metastable Chaos: The Transition to Sustained Chaotic Behavior in the Lorenz Model // Journal of Statistical Physics, Vol. 21, Iss. 3, pp. 263-277. doi:10.1007/BF01011469

[7] C. Sparrow (1982), "The Lorenz equations: bifurcations, chaos, and strange attractors", Springer, New York, 269 pp. doi:10.1007/978-1-4612-5767-7

[8] D. A. Kaloshin (2001), Search for and Stabilization of Unstable Saddle Cycles in the Lorenz System // Differential Equations, Vol. 37, Iss. 11, pp. 1636-1639. doi:10.1023/A:1017933202944

[9] L.-S. Yao (2010), Computed Chaos or Numerical Errors // Nonlinear Analysis: Modelling and Control, Vol. 15, Iss. 1, pp. 109-126. http://www.mii.1t/na/issues/NA_1501/NA15109.pdf

[10] I. Babuska, M. Prager, and E. Vitasek (1966), "Numerical processes in differential equations", SNTL, Praha; Interscience Publishers John Wiley \& Sons, London - New York - Sydney, 351 pp.

[11] J. Teixeira, C. A. Reynolds, and K. Judd (2007), Time Step Sensitivity of Nonlinear Atmospheric Models: Numerical Convergence, Truncation Error Growth, and Ensemble Design // Journal of the Atmospheric Sciences, Vol. 64, Iss. 1, pp. 175-189. doi:10.1175/JAS3824.1

[12] S. H. Strogatz (1994), "Nonlinear dynamics and chaos, with applications to physics, biology, chemistry and engineering", Perseus Books Publishing, 498 pp.

[13] S. A. Sarra, and C. Meador (2011), On the Numerical Solution of Chaotic Dynamical Systems Using Extend Precision Floating Point Arithmetic and Very High Order Numerical Methods // Nonlinear Analysis: Modelling and Control, Vol. 16, Iss. 3, pp. 340-352. http: //www. lana.1t/journal/42/NA16306.pdf 
[14] E. Hairer, S. P. Norsett, and G. Wanner (1993), "Solving ordinary differential equations I: nonstiff problems", Springer, Berlin, 528 pp. doi:10.1007/978-3-540-78862-1

[15] J. C. Butcher (2003), "Numerical methods for ordinary differential equations", John Wiley \& Sons, Chichester, 425 pp.

[16] S. S. Motsa, P. Dlamini, and M. Khumalo (2013), A New Multistage Spectral Relaxation Method for Solving Chaotic Initial Value Systems // Nonlinear Dynamics, Vol. 72, Iss. 1, pp. 265-283. doi:10.1007/s11071-012-0712-8

[17] S. S. Motsa (2012), A New Piecewise-Quasilinearization Method for Solving Chaotic Systems of Initial Value Problems // Central European Journal of Physics, Vol. 10, Iss. 4, pp. 936-946. doi:10.2478/s11534-011-0124-2

[18] I. Hashim, M. S. M. Noorani, R. Ahmad, S. A. Bakar, E. S. Ismail, and A. M. Zakaria (2006), Accuracy of the Adomian Decomposition Method Applied to the Lorenz System // Chaos, Solitons and Fractals, Vol. 28, Iss. 5, pp. 1149-1158. doi: $10.1016 /$ j.chaos.2005.08.135

[19] O. Abdulaziz, N. F. M. Noor, I. Hashim, and M. S. M. Noorani (2008), Further Accuracy Tests on Adomian Decomposition Method for Chaotic Systems // Chaos, Solitons and Fractals, Vol. 36, Iss. 5, pp. 1405-1411. doi:10.1016/j.chaos.2006.09.007

[20] M. M. Al-Sawalha, M. S. M. Noorani, and I. Hashim (2009), On Accuracy of Adomian Decomposition Method for Hyperchaotic Rössler System // Chaos, Solitons and Fractals, Vol. 40, Iss. 4, pp. 1801-1807. doi:10.1016/j.chaos.2007.09.062

[21] P. Vadasz, and S. Olek (2000), Convergence and Accuracy of Adomian's Decomposition Method for the Solution of Lorenz Equations // International Journal of Heat and Mass Transfer, Vol. 43, Iss. 10, pp. 1715-1734. doi:10.1016/S0017-9310(99)00260-4

[22] A. N. Pchelintsev (2014), Numerical and Physical Modeling of the Dynamics of the Lorenz System // Numerical Analysis and Applications, Vol. 7, Iss. 2, pp. 159-167. 
doi:10.1134/S1995423914020098

[23] R. Lozi, and A. N. Pchelintsev (2015), A New Reliable Numerical Method for Computing Chaotic Solutions of Dynamical Systems: The Chen Attractor Case // International Journal of Bifurcation and Chaos, Vol. 25, Iss. 13, 1550187, 10 pp. doi:10.1142/S0218127415501874

[24] G. Chen, and T. Ueta (1999), Yet Another Chaotic Attractor // International Journal of Bifurcation and Chaos, Vol. 9, Iss. 7, pp. 1465-1466. doi:10.1142/S0218127499001024

[25] T. Ueta, and G. Chen (2000), Bifurcation Analysis of Chen's Attractor // International Journal of Bifurcation and Chaos, Vol. 10, Iss. 8, pp. 1917-1931.

doi:10.1142/S0218127400001183

[26] W. G. Hoover (1985), Canonical Dynamics: Equilibrium Phase-Space Distributions // Physical Review A, Vol. 31, Iss. 3, 1695. doi:10.1103/PhysRevA.31.1695

[27] S. Jafari, J. C. Sprott, and F. Nazarimehr (2015), Recent New Examples of Hidden Attractors // The European Physical Journal Special Topics, Vol. 224, Iss. 8, pp. 1469-1476. doi:10.1140/epjst/e2015-02472-1

[28] J. C. Sprott (1994), Some Simple Chaotic Flows // Physical Review E, Vol. 50, Iss. 2, R647. doi:10.1103/PhysRevE.50.R647

[29] R. Barrio, M. A. Martínez, S. Serrano, and D. Wilczak (2015), When Chaos Meets Hyperchaos: 4D Rössler Model // Physics Letters A, Vol. 379, Iss. 38, pp. 2300-2305. doi:10.1016/j.physleta.2015.07.035

[30] P. Reitererdag, C. Lainscsekdag, F. Schürrerdag, C. Letellierddag, and J. Maquet (1998), A Nine-Dimensional Lorenz System to Study HighDimensional Chaos // Journal of Physics A: Mathematical and General, Vol. 31, pp. 7121-7139. doi:10.1088/0305-4470/31/34/015 
[31] A. Algaba, F. Fernández-Sánchez, M. Merino, and A. J. RodríguezLuis (2013), Chen's Attractor Exists if Lorenz Repulsor Exists: The Chen System is a Special Case of the Lorenz System // Chaos, Vol. 23, 033108 .

doi: $10.1063 / 1.4813227$

[32] G. Chen (2013), The Chen System Revisited // Dynamics of Continuous, Discrete and Impulsive Systems Series B: Applications and Algorithms, Vol. 20, pp. 691-696.

http://www.ee.cityu.edu.hk/ gchen/pdf/Chen_Sys_Revited_ 2013.pdf 